\title{
Research Article \\ Infra Soft Compact Spaces and Application to Fixed Point Theorem
}

\author{
Tareq M. Al-shami \\ Department of Mathematics, Sana'a University, Sana'a, Yemen \\ Correspondence should be addressed to Tareq M. Al-shami; tareqalshami83@gmail.com
}

Received 12 June 2021; Accepted 2 July 2021; Published 15 July 2021

Academic Editor: Huseyin Isik

Copyright ( 2021 Tareq M. Al-shami. This is an open access article distributed under the Creative Commons Attribution License, which permits unrestricted use, distribution, and reproduction in any medium, provided the original work is properly cited.

\begin{abstract}
Infra soft topology is one of the recent generalizations of soft topology which is closed under finite intersection. Herein, we contribute to this structure by presenting two kinds of soft covering properties, namely, infra soft compact and infra soft Lindelöf spaces. We describe them using a family of infra soft closed sets and display their main properties. With the assistance of examples, we mention some classical topological properties that are invalid in the frame of infra soft topology and determine under which condition they are valid. We focus on studying the "transmission" of these concepts between infra soft topology and classical infra topology which helps us to discover the behaviours of these concepts in infra soft topology using their counterparts in classical infra topology and vice versa. Among the obtained results, these concepts are closed under infra soft homeomorphisms and finite product of soft spaces. Finally, we introduce the concept of fixed soft points and reveal main characterizations, especially those induced from infra soft compact spaces.
\end{abstract}

\section{Introduction}

In our daily life, we face many types of uncertain phenomena and problems which require looking for adequate approaches to deal with them. The researchers' efforts in this regard lead to proposing various convenient tools to address uncertainty and vagueness. One of the notable tools related to our interest is the soft set which was introduced in 1999 by Molodtsov [1]. He discussed its different applications like smoothness of functions, game theory, theory of measurement, and Riemann integration. Then, soft sets have been applied to several scopes like medical science [2], computer science [3], and decision-making problems [4].

After three years of the emergence of soft sets, Maji with his coauthors [5] put forward the basic concepts of soft set theory. They explored primary operations like the intersection of two soft sets and their union and difference; also, they defined a complement of soft set. Later on, many scholars and researchers interested in soft set theory redefined the concepts given by Maji et al. and displayed new types of soft operations and operators. To keep some properties and results of crisp set theory, Ali et al. [6] proposed new types of these operations and operators. The improvements and contributions to soft set theory have been continued which lead to defining many sorts of soft equality like lower and upper soft equality [7], $g f$-soft equality [8], and $T$-soft equality [9].

As it is well known, topologists employed the generalizations of crisp sets to construct novel extensions of topologies. As a continuation of this path, Shabir and Naz [10] and Çağ man et al. [11] hybridized classical topology and soft sets to formulate soft topological spaces. In fact, they differently formulated the concept of soft topology. Shabir and Naz stipulated the constant of the universal crisp set and set of parameters which the members of soft topology defined over them, whereas Çağman et al. did not impose any conditions on the universal crisp set and set of parameters. Our approach in this paper goes according to the definition of Shabir and Naz. Special kinds of soft topologies called enriched and extended soft topologies were introduced in [12]. Al-Shami and Kocinac [13] scrutinized the role of extended soft topology to link the concepts in soft topologies with their counterparts in classical topologies.

It was noted that several properties of topological concepts are preserved under some generalizations of soft 
topology such as supra soft topology [14], soft bitopology [15], and infra soft topology [16]. This means that we can consider that some topology's conditions are superfluous in some cases. In fact, this matter was applied in crisp settings to describe some real-life issues (see $[17,18]$ ). Another merit of generalizations of soft topology is the ease of building counterexamples that show the relationships between the concepts under study. For these reasons, we are interested to study infra soft topology which is one of the recent interesting developments of soft topology.

Compactness and Lindelöfness are one of the interesting concepts in soft topologies. They were studied in some pioneer articles such as [19-21]. Our contribution herein is to analyze the properties of these two concepts in the frame of infra soft topological spaces. We note the validity of some properties of (classical) soft compactness and Lindelöf spaces via infra soft topological structures. This helps us to discuss many topological concepts and reveal the relationships among them in this frame instead of soft topology, so we target in this manuscript to perform an exhaustive analysis of infra soft topological structures.

The fixed point theorem is a hot topic in recent years. It has been investigated in many papers such as [22-25]. In this work, we put forward the basis of fixed soft points in the introduced frame. Some results that associated fixed soft points with infra soft compact spaces and infra parametric infra topological spaces are studied in detail.

The layout followed in the rest of this manuscript is as follows. In Section 2, we recall the definitions and results that we need to comprehend this work. In Section 3, we introduce the concepts of infra soft compact and infra soft Lindelöf spaces and characterize them. We establish their master features and reveal some of their counterparts' properties that are lost. In Section 4, we study fixed soft points in the frame of infra soft topological spaces and explore the role of infra soft compactness to obtain a fixed soft point. Finally, we epitomize the paper's fulfillments and suggest some future works in Section 5.

\section{Preliminaries}

Through this section, we mention the materials that make this study self-contained. We divide it into two subsections.

\subsection{Soft Set Theory}

Definition 1 (see [1]). Let $\mathscr{B}$ be a set of parameters, $\mathscr{A}$ a universal set, and $2^{\mathscr{A}}$ the power set of $\mathscr{A}$. A soft set over $\mathscr{A}$ is an ordered pair $(\delta, \mathscr{B})$, where $\delta: \mathscr{B} \longrightarrow 2^{\mathscr{A}}$ is a crisp map. We express the soft set as follows: $(\delta, \mathscr{B})=\{(\beta, \delta(\beta)): \beta \in \mathscr{B}$ and $\left.\delta(\beta) \in 2^{\mathscr{A}}\right\}$.

A family of all soft sets over $\mathscr{A}$ under a set of parameters $\mathscr{B}$ is symbolized by $S\left(\mathscr{A}_{\mathscr{B}}\right)$.

Definition 2 (see [6]). A soft set $\left(\delta^{c}, \mathscr{B}\right)$ is called a complement of $(\delta, \mathscr{B})$ provided that a map $\delta^{c}: \mathscr{B} \longrightarrow 2^{\mathscr{A}}$ such that $\delta^{c}(\beta)=\mathscr{A} \backslash \delta(\beta)$ for each $\beta \in \mathscr{B}$.
Definition 3 (see [5]). If the image of each parameter of $\mathscr{B}$ under a map $\delta: \mathscr{B} \longrightarrow 2^{\mathscr{A}}$ is the universal set $\mathscr{A}$, then $(\delta$, $\mathscr{B})$ is called the absolute soft set over $\mathscr{A}$. Its complement is called the null soft set. The absolute and null soft sets are symbolized by $\tilde{\mathscr{A}}$ and $\Phi$, respectively.

Definition 4 (see $[26,27])$. If all components of a soft set are equal (resp., finite, countable), then we called it a stable (resp., finite, countable) soft set. Otherwise, it is called unstable (resp., infinite, uncountable).

Definition 5 (see $[12,27]$ ). If the image of one parameter, say $\beta$, under a map $P: \mathscr{B} \longrightarrow 2^{\mathscr{A}}$ is a singleton set, say $\{\alpha\}$, and the image of each parameter $\beta^{\prime} \in \mathscr{B} \backslash\{\beta\}$ is the empty set, then a soft set $(P, \mathscr{B})$ is called a soft point over $\mathscr{A}$. It is briefly symbolized by $P_{\beta}^{\alpha}$.

Definition 6 (see $[10,27])$. There are two belong and two nonbelong relations between an element $\alpha \in \mathscr{A}$ and a soft set $(\delta, \mathscr{B})$ defined as follows.

(i) $\alpha \in(\delta, \mathscr{B})$ if $\alpha \in \delta(\beta)$ for all $\beta \in \mathscr{B}$

(ii) $\alpha \in(\delta, \mathscr{B})$ if $\alpha \in \delta(\beta)$ for some $\beta \in \mathscr{B}$

(iii) $\alpha \Subset(\delta, \mathscr{B})$ if $\alpha \in \delta(\beta)$ for some $\beta \in \mathscr{B}$

(iv) $\alpha \Subset(\delta, \mathscr{B})$ if $\alpha \in \delta(\beta)$ for all $\beta \in \mathscr{B}$

Definition 7 (see [6]). The intersection of two soft sets $(\delta, \mathscr{B})$ and $(\xi, \mathscr{C})$ over $\mathscr{A}$, symbolized by $(\delta, \mathscr{B}) \bigcap^{\sim}(\xi, \mathscr{C})$, is a soft set $(\lambda, \mathscr{D})$, where $\mathscr{D}=\mathscr{B} \cap \mathscr{C} \neq \varnothing$, and a map $\lambda: \mathscr{D} \longrightarrow 2^{\mathscr{A}}$ is given by $\lambda(\beta)=\delta(\beta) \cap \xi(\beta)$ for each $\beta \in \mathscr{D}$.

Definition 8 (see [5]). The union of two soft sets $(\delta, \mathscr{B})$ and $(\xi, \mathscr{C})$ over $\mathscr{A}$, symbolized by $(\delta, \mathscr{B}) \bigcup^{\sim}(\xi, \mathscr{C})$, is a soft set $(\lambda, \mathscr{D})$, where $\mathscr{D}=\mathscr{B} \cup \mathscr{C}$ and a map $\lambda: \mathscr{D} \longrightarrow 2^{\mathscr{A}}$ is given as follows:

$$
\lambda(\beta)= \begin{cases}\delta(\beta) & : \beta \in \mathscr{B} \backslash \mathscr{C}, \\ \xi(\beta) & : \beta \in \mathscr{C} \backslash \mathscr{B}, \\ \delta(\beta) \cup \xi(\beta) & : \beta \in \mathscr{B} \cap \mathscr{C} .\end{cases}
$$

Definition 9 (see [28]). A soft set $(\delta, \mathscr{B})$ is a subset of a soft set $(\xi, \mathscr{C})$, symbolized by $(\delta, \mathscr{B}) \subseteq \sim(\xi, \mathscr{C})$, if $\mathscr{B} \subseteq \mathscr{C}$ and $\delta(\beta)$ $\subseteq \xi(\beta)$ for all $\beta \in \mathscr{B}$. The soft sets $(\delta, \mathscr{B})$ and $(\xi, \mathscr{C})$ are called soft equal if each is a subset of the other.

Definition 10 (see [19]). A family of soft sets is said to have the finite (resp., countable) intersection property if the finite (resp., countable) intersection of any members of this family is nonnull.

Definition 11 (see [20]). The Cartesian product of $(\delta, \mathscr{B})$ and $(\xi, \mathscr{C})$, symbolized by $(\delta \times \xi, \mathscr{B} \times \mathscr{C})$, is defined as $(\delta \times \xi)$ $\left(\beta, \beta^{\prime}\right)=\delta(\beta) \times \xi\left(\beta^{\prime}\right)$ for each $\left(\beta, \beta^{\prime}\right) \in \mathscr{B} \times \mathscr{C}$. 
Definition 12 (see [29]). A soft map $f_{\varphi}$ from $S\left(\mathscr{A}_{\mathscr{B}}\right)$ to $S\left(\mathscr{Z}_{\mathscr{C}}\right)$ is a pair of crisp maps $f$ and $\varphi$, where $f: \mathscr{A} \longrightarrow \mathscr{Z}, \varphi: \mathscr{B}$ $\longrightarrow \mathscr{C}$. Let $(\delta, \mathscr{M})$ and $(\xi, \mathscr{N})$ be, respectively, subsets of $S\left(\mathscr{A}_{\mathscr{B}}\right)$ and $S\left(\mathscr{Z}_{\mathscr{C}}\right)$. Then, the image of $(\delta, \mathscr{M})$ and preimage of $(\xi, \mathcal{N})$ are given by the following.

(i) $f_{\varphi}(\delta, \mathscr{M})=(f(\delta), \mathscr{C})$ is a soft set in $S\left(\mathscr{X}_{\mathscr{C}}\right)$ such that

$$
f(\delta)(\omega)= \begin{cases}\widetilde{\bigcup}_{\beta \in \varphi^{-1}(\omega) \cap \mathscr{M}} f(\delta(\beta)) & : \varphi^{-1}(\omega) \neq \varnothing, \\ \varnothing & : \varphi^{-1}(\omega)=\varnothing,\end{cases}
$$

for each $\omega \in \mathscr{C}$

(ii) $f_{\varphi}^{-1}(\xi, \mathscr{N})=\left(f^{-1}(\xi), \mathscr{B}\right)$ is a soft set in $S\left(\mathscr{A}_{\mathscr{B}}\right)$ such that

$$
f^{-1}(\xi)(\beta)= \begin{cases}f^{-1}(\xi(\varphi(\beta))) & : \varphi(\beta) \in \mathcal{N}, \\ \varnothing & : \varphi(\beta) \in \mathcal{N},\end{cases}
$$

for each $\beta \in \mathscr{B}$

Definition 13 (see [20, 30]). A soft map $f_{\varphi}: S\left(\mathscr{A}_{\mathscr{B}}\right) \longrightarrow S$ $\left(\mathscr{Z}_{\mathscr{C}}\right)$ is said to be injective (resp., surjective, bijective) if both $f$ and $\varphi$ are injective (resp., surjective, bijective).

\subsection{Infra Soft Topological Spaces}

Definition 14 (see [16]). A family $\Omega$ of soft sets over $\mathscr{A}$ with $\mathscr{B}$ as a parameter set is said to be an infra soft topology on $\mathscr{A}$ if it is closed under finite intersection and $\Phi$ is a member of $\Omega$.

The triple $(\mathscr{A}, \Omega, \mathscr{B})$ is called an infra soft topological space (briefly, ISTS). We called a member of $\Omega$ an infra soft open set and called its complement an infra soft closed set. We called $(\mathscr{A}, \Omega, \mathscr{B})$ stable if all its infra soft open sets are stable and called finite (resp., countable) if $\mathscr{A}$ is finite (resp., countable).

Proposition 15 (see [16]). Let $(\mathscr{A}, \Omega, \mathscr{B})$ be an ISTS. Then, the collection $\Omega_{\beta}=\{\delta(\beta):(\delta, \mathscr{B}) \in \Omega\}$ forms an infra topology on $\mathscr{A}$ for each $\beta \in \mathscr{B}$.

We called $\Omega_{\beta}$ a parametric infra topology.

Proposition 16 (see [16]). Suppose that $\Psi=\left\{\Omega_{\beta}\right\}_{\beta \in \mathscr{B}}$ is a class of crisp infra topologies on $\mathscr{A}$. Then,

$\Omega(\Psi)=\left\{\{(\beta, F(\beta)): \beta \in \mathscr{B}\} \in S\left(\mathscr{A}_{\mathscr{B}}\right)\right.$ such that $F(\beta) \in \Omega_{\beta}$ for each $\left.\beta \in \mathscr{B}\right\}$

defines an infra soft topology on $\mathscr{A}$.
The ISTS given in the above proposition is called an extended infra soft topology on $\mathscr{A}$ or an infra soft topology on $\mathscr{A}$ generated by $\Psi$.

Definition 17. An ISTS $(\mathscr{A}, \Omega, \mathscr{B})$ is called an infra $p p$-soft $\mathrm{T}_{2}$ (or an infra $p p$-soft Hausdorff)-space if for every $\alpha \neq \varepsilon \in$ $\mathscr{A}$, there exist disjoint infra soft open sets $(\delta, \mathscr{B})$ and $(\xi, \mathscr{B})$ such that $\alpha \Subset(\delta, \mathscr{B}), \varepsilon \Subset(\xi, \mathscr{B})$ and $\varepsilon \in(\delta, \mathscr{B}), \alpha \in(\xi, \mathscr{B})$.

In the above definition, if we replace the relations $(\Subset, \epsilon)$ by $(\Subset, \Subset)$ (resp., $(\epsilon, \epsilon)(\epsilon, \Subset)$ ), then we called $(\mathscr{A}, \Omega, \mathscr{B})$ an infra $p t$-soft $T_{2}$ (resp., infra $t p$-soft $T_{2}$, infra $t t$-soft $T_{2}$ )-space.

Definition 18 (see [16]). Let $(\mathscr{A}, \Omega, \mathscr{B})$ be an ISTS and $(\xi, \mathscr{B})$ be a nonnull subset of $\tilde{\mathscr{A}}$. Then, $\Omega_{(\xi, \mathscr{B})}=\{(\xi, \mathscr{B})$ $\left.\bigcap^{\sim}(\delta, \mathscr{B}):(\delta, \mathscr{B}) \in \Omega\right\}$ is called an infra soft relative topology on $(\xi, \mathscr{B})$ and $\left((\xi, \mathscr{B}), \Omega_{(\xi, \mathscr{B})}, \mathscr{B}\right)$ is called an infra soft subspace of $(\mathscr{A}, \Omega, \mathscr{B})$.

Theorem 19 (see [16]). Let $\left((\xi, \mathscr{B}), \Omega_{(\xi, \mathscr{B})}, \mathscr{B}\right)$ be an infra soft subspace of $(\mathscr{A}, \Omega, \mathscr{B})$. Then, $(\lambda, \mathscr{B})$ is an infra soft closed subset of $\left((\xi, \mathscr{B}), \Omega_{(\xi, \mathscr{B})}, \mathscr{B}\right)$ iff there exists an infra soft closed subset $(\delta, \mathscr{B})$ of $(\mathscr{A}, \Omega, \mathscr{B})$ such that $(\lambda, \mathscr{B})=(\xi, \mathscr{B})$ $\bigcap^{\sim}(\delta, \mathscr{B})$.

Definition 20 (see [31]). A soft mapping $f_{\varphi}:\left(\mathscr{A}_{1}, \Omega_{1}, \mathscr{B}_{1}\right)$ $\longrightarrow\left(\mathscr{A}_{2}, \Omega_{2}, \mathscr{B}_{2}\right)$ is said to be

(i) infra soft continuous provided that the preimage of any infra soft open set is an infra soft open set

(ii) infra soft open (resp., infra soft closed) if the image of any infra soft open (resp., infra soft closed) set is an infra soft open (resp., infra soft closed) set

(iii) an infra soft homeomorphism if it is infra soft continuous, infra soft open, and bijective

A property which is kept by any infra soft homeomorphism is said to be an infra soft topological property.

Proposition 21 (see [31]). Let $\left\{\left(\mathscr{A}_{k}, \Omega_{k}, \mathscr{B}_{k}\right): k \in K\right\}$ be a family of ISTSs. Then, $\Omega=\left\{\prod_{k \in K}\left(\delta_{k}, \mathscr{B}_{k}\right):\left(\delta_{k}, \mathscr{B}_{k}\right) \in \tau_{k}\right\}$ is an infra soft topology on $\mathscr{A}=\prod_{k \in K} \mathscr{A}_{k}$ under a set of parameters $\mathscr{B}=\prod_{k \in K} \mathscr{B}_{k}$.

We called $\Omega$ given in the proposition above a product of infra soft topologies and $(\mathscr{A}, \Omega, \mathscr{B})$ a product of infra soft spaces.

\section{Infra Soft Compact and Infra Soft Lindelöf Spaces}

This section is devoted to investigating compactness and Lindelöfness in infra soft topological spaces. We scrutinize their main properties and bring to light some celebrated results of classical compactness and Lindelöfness that are invalid in the 
frame of ISTS. For illustration and validation, various examples are offered.

\section{Definition 22.}

(i) A family of infra soft open sets $\left\{\left(\delta_{k}, \mathscr{B}\right): k \in K\right\}$ is said to be an infra soft open cover of an ISTS $(\mathscr{A}$, $\Omega, \mathscr{B})$ provided that the union of its elements covers $\tilde{\mathscr{A}}$, i.e., $\tilde{\mathscr{A}}=\bigcup^{\sim}{ }_{k \in K}\left(\delta_{k}, \mathscr{B}\right)$

(ii) An ISTS $(\mathscr{A}, \Omega, \mathscr{B})$ is said to be infra soft compact (resp., infra soft Lindelöf) provided that every infra soft open cover of $\tilde{\mathscr{A}}$ has a finite (resp., countable) subcover

(iii) A soft subset $(\delta, \mathscr{B})$ of an ISTS $(\mathscr{A}, \Omega, \mathscr{B})$ is said to be infra soft compact (resp., infra soft Lindelöf) provided that every infra soft open cover of $(\delta, \mathscr{B})$ has a finite (resp., countable) subcover

By the two examples below, we explain the existence and uniqueness of infra soft compact and infra soft Lindelöf spaces.

Example 23. Let $\Omega=\{\widetilde{\mathbb{R}}\} \bigcup\{(\delta, \mathscr{B}) \subseteq \sim \widetilde{\mathbb{R}}: 3 \Subset(\delta, \mathscr{B})\}$ be an infra soft topology on the real number set $\mathbb{R}$ with $\mathscr{B}$ as an arbitrary set of parameters. It is easy to check that $(\mathbb{R}, \Omega, \mathscr{B})$ is infra soft compact.

Example 24. Let $\Omega=\{\widetilde{\mathbb{R}}\} \bigcup\{(\delta, \mathscr{B}) \subseteq \sim \widetilde{\mathbb{R}}: 3 \in(\delta, \mathscr{B})\}$ be an infra soft topology on the real number set $\mathbb{R}$ with $\mathscr{B}$ as an arbitrary set of parameters such that $|\mathscr{B}| \geq 2$. Now, the family $\{(\delta, \mathscr{B}) \subseteq \sim \widetilde{\mathbb{R}}$ : there is only one parameter $\beta \in \mathscr{B}$ such that 3 $\epsilon \delta(\beta)\}$ forms an infra soft open cover of $\widetilde{\mathbb{R}}$. It is easy to check that this infra soft open cover does not have a countable subcover; hence, $(\mathbb{R}, \Omega, \mathscr{B})$ is not infra soft Lindelöf.

The proofs of the next two results are easy, so they will be canceled.

Proposition 25. Every infra soft compact space is infra soft Lindelöf.

Proposition 26. A family of infra soft compact (resp., infra soft Lindelöf) sets is closed under a finite (resp., countable) union.

By the example below, we explain that Proposition 25 is not converse.

Example 27. A family $\Omega=\{\widetilde{\mathbb{N}},(\delta, \mathscr{B}) \subseteq \sim \widetilde{\mathbb{N}}:(\delta, \mathscr{B})$ is finite $\}$ represents an infra soft topology on the set of natural numbers $\mathbb{N}$ with $\mathscr{B}=\left\{\beta_{1}, \beta_{2}\right\}$ as a set of parameters. It is easy to check that $(\mathbb{N}, \Omega, \mathscr{B})$ is an infra soft Lindelöf space, but not infra soft compact.
Proposition 28. Every infra soft closed subset $(\xi, \mathscr{B})$ of an infra soft compact (resp., infra soft Lindelöf) space $(\mathscr{A}, \Omega, \mathscr{B})$ is infra soft compact (resp., infra soft Lindelöf).

Proof. Consider $\left\{\left(\delta_{k}, \mathscr{B}\right): k \in K\right\}$ as an infra soft open cover of $(\xi, \mathscr{B})$ which is a subset of an infra soft compact space $(\mathscr{A}, \Omega, \mathscr{B})$. Then, $\bigcup_{k \in K}^{\sim}\left(\delta_{k}, \mathscr{B}\right) \bigcup^{\sim}\left(\xi^{c}, \mathscr{B}\right)$ is an infra soft open cover of $\tilde{\mathscr{A}}$. By hypothesis, $\tilde{\mathscr{A}}=\bigcup_{k=1}^{\sim n}\left(\delta_{k}, \mathscr{B}\right)$ $\bigcup^{\sim}\left(\xi^{c}, \mathscr{B}\right)$. Consequently, we obtain $(\xi, \mathscr{B}) \subseteq \sim \cup_{k=1}^{\sim n}\left(\delta_{k}, \mathscr{B}\right)$. This ends the proof that $(\xi, \mathscr{B})$ is infra soft compact.

Following a similar technique, one can prove the case between parentheses.

The converse of the above proposition fails as illustrated in the next example.

Example 29. Consider an ISTS $(\mathbb{R}, \Omega, \mathscr{B})$ as given in Example 23. Let $\mathscr{B}=\left\{\beta_{1}, \beta_{2}\right\}$. Then, $(\delta, \mathscr{B})=\left\{\left(\beta_{1},\{5\}\right),\left(\beta_{2},\{5\}\right)\right\}$ is infra soft compact, but not infra soft closed.

Corollary 30. The intersection of infra soft compact (resp., infra soft Lindelöf) and infra soft closed sets is infra soft compact (resp., infra soft Lindelöf).

One of the celebrated results in classical topology reports that the finite (resp., countable) topological space is compact (resp., Lindelöf); this result evaporates in ISTSs as the next example elucidates.

Example 31. Let $\Omega_{1}$ and $\Omega_{2}$ be two discrete infra soft topologies on a finite set $\mathscr{A}_{1}$ and a countable set $\mathscr{A}_{2}$, respectively. Let the sets of natural numbers $\mathbb{N}$ and real numbers $\mathbb{R}$ be sets of parameters. It is clear that $\left(\mathscr{A}_{1}, \Omega_{1}, \mathbb{N}\right)$ is not infra soft compact in spite of $\mathscr{A}_{1}$ being finite, and $\left(\mathscr{A}_{2}, \Omega_{2}, \mathbb{R}\right)$ is not infra soft Lindelöf in spite of $\mathscr{A}_{2}$ being countable.

Note that the intersection of two infra soft compact (resp., infra soft Lindelöf) sets needs not be infra soft compact (resp., infra soft Lindelöf). The example given below confirms this fact.

Example 32. Consider an ISTS $(\mathbb{R}, \Omega, \mathscr{B})$ as given in Example 23. Let $\mathscr{B}=\left\{\beta_{1}, \beta_{2}\right\}$. Note that the two soft sets $\left(\delta_{1}, \mathscr{B}\right)$ $=\left\{\left(\beta_{1}, \mathbb{R} \backslash\{3\}\right),\left(\beta_{2}, \mathbb{R}\right)\right\}$ and $\left(\delta_{2}, \mathscr{B}\right)=\left\{\left(\beta_{1}, \mathbb{R}\right),\left(\beta_{2}, \mathbb{R} \backslash\right.\right.$ $\{3\})\}$ are infra soft compact. But their intersection is the soft set $\left\{\left(\beta_{1}, \mathbb{R} \backslash\{3\}\right),\left(\beta_{2}, \mathbb{R} \backslash\{3\}\right)\right\}$ which is not infra soft Lindelöf.

Now, we give a complete description for infra soft compact and infra soft Lindelöf spaces using a family of infra soft closed sets.

Theorem 33. An ISTS $(\mathscr{A}, \Omega, \mathscr{B})$ is infra soft compact (resp., infra soft Lindelöf) iff every family of infra soft closed subsets of $(\mathscr{A}, \Omega, \mathscr{B})$, satisfying the finite (resp., countable) intersection property, has, itself, a nonnull intersection. 
Proof. Necessity: consider $\Sigma=\left\{\left(\delta_{k}, \mathscr{B}\right): k \in K\right\}$ as a family of infra soft closed subsets of $(\mathscr{A}, \Omega, \mathscr{B})$ which is an infra soft compact space. Suppose that $\bigcap_{k \in K}^{\sim}\left(\delta_{k}, \mathscr{B}\right)=\Phi$. Then, $\tilde{\mathscr{A}}=$ $\bigcup_{k \in K}^{\sim}\left(\delta_{k}^{c}, \mathscr{B}\right)$. By the hypothesis of infra soft compactness, we obtain $\tilde{\mathscr{A}}=\bigcup_{k=1}^{\sim n}\left(\delta_{k}^{c}, \mathscr{B}\right)$. Hence, $\bigcap_{k=1}^{n}\left(\delta_{k}, \mathscr{B}\right)=\Phi$, as required.

Sufficiency: consider $\Sigma=\left\{\left(\delta_{k}, \mathscr{B}\right): k \in K\right\}$ as an infra soft open cover of $(\mathscr{A}, \Omega, \mathscr{B})$. Suppose that $\Sigma$ does not have a finite subcover of $\tilde{\mathscr{A}}$. Then $\tilde{\mathscr{A}} \backslash \bigcup_{k \in I}\left(\delta_{k}, \mathscr{B}\right) \neq \Phi$ for any finite set $I \subseteq K$. Therefore, $\bigcap_{k \in I}^{\sim}\left(\delta_{k}^{c}, \mathscr{B}\right) \neq \Phi$. This means that $\left\{\left(\delta_{k}^{c}, \mathscr{B}\right): k \in I\right\}$ is a family of infra soft closed subsets of $\tilde{\mathscr{A}}$ which has the finite intersection property. Thus, $\bigcap_{k \in K}^{\sim}\left(\delta_{k}^{c}, \mathscr{B}\right) \neq \Phi$. Consequently, $\tilde{\mathscr{A}} \neq \bigcup^{\sim}{ }_{k \in I}\left(\delta_{k}, \mathscr{B}\right)$. But this contradicts that $\Sigma$ is an infra soft open cover of $\tilde{\mathscr{A}}$. Hence, $(\mathscr{A}, \Omega, \mathscr{B})$ is infra soft compact.

Following a similar technique, one can prove the case between parentheses.

In the next example, we show that there is no relationship between infra soft closed sets and the different types of infra soft $T_{2}$-spaces.

Example 34. Consider an ISTS $(\mathscr{N}, \Omega, \mathscr{B})$ as given in Example 27. Note that a soft set $\left(\delta_{1}, \mathscr{B}\right)=\left\{\left(\beta_{1}, \mathbb{N} \backslash\{1, \sqrt{2}\}\right),\left(\beta_{2}\right.\right.$ $, \mathbb{N} \backslash\{2, \sqrt{3}\})\}$ is infra soft closed. But it is not infra soft compact in spite of $(\mathcal{N}, \Omega, \mathscr{B})$ being an infra $t t$-soft $T_{2}$ (infra tp -soft $T_{2}$, infra pt -soft $T_{2}$, infra $p p$-soft $\left.T_{2}\right)$-space.

Now, we investigate under which conditions the wellknown relationship between closed sets and $T_{2}$-spaces are satisfied in the frame of ISTSs.

Proposition 35. Let $(\mathscr{A}, \Omega, \mathscr{B})$ be an infra tt-soft Hausdorff space such that $\Omega$ is closed under a finite union. Then, every stable infra soft compact subset of $(\mathscr{A}, \Omega, \mathscr{B})$ is infra soft closed.

Proof. Let $(\xi, \mathscr{B})$ be a stable infra soft compact subset of an infra $t$-soft Hausdorff space $(\mathscr{A}, \Omega, \mathscr{B})$, and let $P_{\beta}^{\alpha} \in$ $(\xi, \mathscr{B})^{c}$. Since $(\xi, \mathscr{B})$ is stable, we get $\alpha \neq \gamma_{k}$ for each $P_{\beta}^{\gamma_{k}} \in$ $(\xi, \mathscr{B})$. Therefore, there are two disjoint infra soft open sets $\left(\delta_{k}, \mathscr{B}\right)$ and $\left(\lambda_{k}, \mathscr{B}\right)$ such that $\alpha \in\left(\delta_{k}, \mathscr{B}\right)$ and $\gamma_{k} \in\left(\lambda_{k}, \mathscr{B}\right)$. Now, $\left\{\left(\lambda_{k}, \mathscr{B}\right): k \in K\right\}$ forms an infra soft open cover of $(\xi$, $\mathscr{B})$. By hypothesis, $(\xi, \mathscr{B}) \subseteq \sim \bigcup_{k=1}^{\sim n}\left(\lambda_{k}, \mathscr{B}\right)$. Since $\Omega$ is an infra soft topology closing under a finite union, $\bigcap_{k=1}^{n}\left(\delta_{k}, \mathscr{B}\right)$ and $\bigcup_{k=1}^{\sim n}\left(\lambda_{k}, \mathscr{B}\right)$ are disjoint infra soft open sets. Note that $P_{\beta}^{\alpha} \in \bigcap_{k=1}^{\sim n}\left(\delta_{k}, \mathscr{B}\right) \subseteq \sim(\xi, \mathscr{B})^{c}$. Thus, $(\xi, \mathscr{B})^{c}$ is infra soft open set which automatically means that $(\xi, \mathscr{B})$ is infra soft closed.

Corollary 36. Let $(\mathscr{A}, \Omega, \mathscr{B})$ be an infra tp-soft Hausdorff space such that $\Omega$ is closed under a finite union. Then every stable infra soft compact subset of $(\mathscr{A}, \Omega, \mathscr{B})$ is infra soft closed.
Corollary 37. Let $(\mathscr{A}, \Omega, \mathscr{B})$ be a stable infra pp-soft Hausdorff space such that $\Omega$ is closed under a finite union. Then every stable infra soft compact subset of $(\mathscr{A}, \Omega, \mathscr{B})$ is infra soft closed.

Theorem 38. Let $(\xi, \mathscr{B})$ be an infra soft compact subset of an infra tt -soft Hausdorff space $(\mathscr{A}, \Omega, \mathscr{B})$ such that $\Omega$ is closed under a finite union. Then for each $\alpha \Subset(\xi, \mathscr{B})$, there are disjoint infra soft open sets $(\delta, \mathscr{B})$ and $(\lambda, \mathscr{B})$ such that $\alpha \in$ $(\delta, \mathscr{B})$ and $(\xi, \mathscr{B}) \subseteq \sim(\lambda, \mathscr{B})$.

Proof. Let $\alpha \Subset(\xi, \mathscr{B})$. Then $\alpha \neq \gamma_{k}$ for each $\gamma_{k} \Subset(\xi, \mathscr{B})$. By hypothesis, there exist infra soft open sets $\left(\delta_{k}, \mathscr{B}\right)$ and $\left(\lambda_{k}\right.$, $\mathscr{B})$ such that $\alpha \in\left(\delta_{k}, \mathscr{B}\right)$ and $\gamma_{k} \in\left(\lambda_{k}, \mathscr{B}\right)$. Now, $\left\{\left(\lambda_{k}, \mathscr{B}\right)\right.$ : $k \in K\}$ forms an infra soft open cover of $(\xi, \mathscr{B})$. By the infra soft compactness of $(\xi, \mathscr{B})$ we obtain $(\xi, \mathscr{B}) \subseteq \bigcup_{k=1}^{\sim n}\left(\lambda_{k}, \mathscr{B}\right)$. Since $\Omega$ is an infra soft topology closing under a finite union, $\bigcup_{k=1}^{n}\left(\lambda_{k}, \mathscr{B}\right)$ and $\bigcap_{k=1}^{\sim n}\left(\delta_{k}, \mathscr{B}\right)$ are the required disjoint infra soft open sets. Hence, the proof is complete.

Theorem 39. Let $(\mathscr{A}, \Omega, \mathscr{B})$ be an infra tt-soft Hausdorff space which is infra soft compact and closed under a finite union. Then it is infra tt-soft regular.

Proof. Let $(\xi, \mathscr{B})$ be an infra soft closed set such that $\alpha \Subset(\xi$, $\mathscr{B})$. Then, $(\xi, \mathscr{B})$ is infra soft compact. According to Theorem 38 , there exist disjoint infra soft open sets $(\delta, \mathscr{B})$ and $(\lambda, \mathscr{B})$ such that $\alpha \in(\delta, \mathscr{B})$ and $(\xi, \mathscr{B}) \subseteq \sim(\lambda, \mathscr{B})$. Hence, $(\mathscr{A}, \Omega, \mathscr{B})$ is infra $t$-soft regular.

Corollary 40. Let $(\mathscr{A}, \Omega, \mathscr{B})$ be an infra tt-soft Hausdorff space which is infra soft compact and closed under a finite union. Then it is infra tt-soft $T_{3}$.

Definition 41. An ISTS $(\mathscr{A}, \Omega, \mathscr{B})$ is said to be infra soft $T_{2}^{\prime}$ -space if for every $P_{\beta_{1}}^{\alpha_{1}} \neq P_{\beta_{2}}^{\alpha_{2}}\left(\alpha_{1} \neq \alpha_{2}\right.$ or $\left.\beta_{1} \neq \beta_{2}\right)$, there are two disjoint infra soft open sets $(\delta, \mathscr{B})$ and $(\lambda, \mathscr{B})$ such that $P_{\beta_{1}}^{\alpha_{1}} \in(\delta, \mathscr{B})$ and $P_{\beta_{2}}^{\alpha_{2}} \in(\lambda, \mathscr{B})$.

Proposition 42. Let $(\mathscr{A}, \Omega, \mathscr{B})$ be an infra soft $T_{2}^{\prime}$-space such that $\Omega$ is closed under a finite union. Then every infra soft compact subset of $(\mathscr{A}, \Omega, \mathscr{B})$ is infra soft closed.

Proof. Let $(\xi, \mathscr{B})$ be an infra soft compact subset of $(\mathscr{A}, \Omega$, $\mathscr{B})$ which is an infra soft $T_{2}^{\prime}$-space. Let $P_{\beta}^{\alpha} \in(\xi, \mathscr{B})^{c}$. Then for each $P_{\rho_{k}}^{\gamma_{k}} \in(\xi, \mathscr{B})$, we get $P_{\rho_{k}}^{\gamma_{k}} \neq P_{\beta}^{\alpha}$. Therefore, there are two disjoint infra soft open sets $\left(\delta_{k}, \mathscr{B}\right)$ and $\left(\lambda_{k}, \mathscr{B}\right)$ such that $P_{\rho_{k}}^{\gamma_{k}} \in\left(\delta_{k}, \mathscr{B}\right)$ and $P_{\beta}^{\alpha} \in\left(\lambda_{k}, \mathscr{B}\right)$. Now, $\left\{\left(\delta_{k}, \mathscr{B}\right): k \in K\right\}$ forms an infra soft open cover of $(\xi, \mathscr{B})$. By hypothesis, $(\xi$, $\mathscr{B}) \subseteq \sim \bigcup_{k=1}^{\sim}\left(\delta_{k}, \mathscr{B}\right)$. Since $\Omega$ is an infra soft topology closing under a finite union, $\bigcap_{k=1}^{\sim n}\left(\lambda_{k}, \mathscr{B}\right)$ and $\bigcup_{k=1}^{\sim n}\left(\delta_{k}, \mathscr{B}\right)$ are disjoint infra soft open sets. Note that $P_{\beta}^{\alpha} \in$ $\bigcap_{k=1}^{\sim n}\left(\lambda_{k}, \mathscr{B}\right) \subseteq \sim(\xi, \mathscr{B})^{c}$. Thus, $(\xi, \mathscr{B})^{c}$ is infra soft open set which automatically means that $(\xi, \mathscr{B})$ is infra soft closed. 
Proposition 43. The infra soft continuous image of an infra soft compact (resp., infra soft Lindelöf) set is infra soft compact (resp., infra soft Lindelöf).

Proof. Consider $f_{\varphi}:(\mathscr{A}, \Omega, \mathscr{B}) \longrightarrow(\mathscr{C}, \mathcal{\mho}, \mathscr{D})$ as an infra soft continuous map and let $(\xi, \mathscr{B})$ be an infra soft compact subset of $\tilde{\mathscr{A}}$. Suppose that $\left\{\left(\delta_{k}, \mathscr{B}\right): k \in K\right\}$ is an infra soft open cover of $f_{\varphi}(\xi, \mathscr{B})$. Then $(\xi, \mathscr{B}) \subseteq \sim \bigcup^{\sim}{ }_{k \in K} f_{\varphi}^{-1}\left(\delta_{k}, \mathscr{B}\right)$ and $f_{\varphi}^{-1}\left(\delta_{k}, \mathscr{B}\right)$ is an infra soft open set for each $k \in K$. By hypotheses of infra soft compactness of $(\xi, \mathscr{B})$, we obtain $(\xi, \mathscr{B}) \subseteq \sim \bigcup^{\sim n}{ }_{k=1}^{n} f_{\varphi}^{-1}\left(\delta_{k}, \mathscr{B}\right)$. Therefore, $f_{\varphi}(\xi, \mathscr{B}) \subseteq \sim \bigcup^{\sim n}{ }_{k=1} f_{\varphi}$ $\left(f_{\varphi}^{-1}\left(\delta_{k}, \mathscr{B}\right)\right) \subseteq \sim \bigcup^{\sim n}{ }_{k=1}\left(\delta_{k}, \mathscr{B}\right)$. This ends the proof that $f_{\varphi}(\xi$, $\mathscr{B})$ is infra soft compact.

Following similar arguments, one can prove the case between parentheses.

Corollary 44. The property of being an infra soft compact (infra soft Lindelöf) space is preserved under an infra soft homeomorphism, i.e., it is an infra soft topological property.

In the next result, we explain that the properties of infra soft compactness and infra soft Lindelöfness transmit from infra soft topology to its parametric infra topologies under an extended condition.

Theorem 45. If $(\mathscr{A}, \Omega, \mathscr{B})$ is an extended infra soft compact (resp., extended infra soft Lindelöf) space, then $\left(\mathscr{A}, \Omega_{\beta}\right)$ is infra compact (resp., infra Lindelöf) for each $\beta \in \mathscr{B}$.

Proof. Let $\left\{H_{k}: k \in K\right\}$ be an infra open cover of $\left(\mathscr{A}, \Omega_{\beta}\right)$. Consider $\Theta=\left\{\left(\delta_{k}, \mathscr{B}\right): k \in K\right\}$ as a family of soft set which is defined as $\delta_{k}(\beta)=H_{k}$ and $\delta_{k}\left(\beta^{\prime}\right)=\mathscr{A}$ for each $\beta^{\prime} \neq \beta$. Since $\Omega$ is extended, $\Theta$ is an infra soft open cover of $\tilde{\mathscr{A}}$. By the hypothesis of infra soft compactness, we obtain $\tilde{\mathscr{A}}=$ $\bigcup_{k=1}^{\sim n}\left(\delta_{k}, \mathscr{B}\right)$. This implies that $\mathscr{A}=\bigcup_{k=1}^{n} \delta_{k}(\beta)=\bigcup_{k=1}^{n} H_{k}$ which proves that $\left(\mathscr{A}, \Omega_{\beta}\right)$ is an infra compact space.

Following similar arguments, one can prove the case between parentheses.

The converse of the theorem above fails as the next example illustrates.

Example 46. Consider ISTSs $\left(\mathscr{A}_{1}, \Omega_{1}, \mathbb{N}\right)$ and $\left(\mathscr{A}_{2}, \Omega_{2}, \mathbb{R}\right)$ as given in Example 31. It is clear that $\left(\mathscr{A}_{1}, \Omega_{1 n}\right)$ is an infra compact space for all $n \in \mathbb{N}$ and $\left(\mathscr{A}_{2}, \Omega_{2 r}\right)$ is an infra Lindelöf space for all $r \in \mathbb{R}$, whereas $\left(\mathscr{A}_{1}, \Omega_{1}, \mathbb{N}\right)$ is not infra soft compact and $\left(\mathscr{A}_{2}, \Omega_{2}, \mathbb{R}\right)$ is not infra soft Lindelöf.

Note that an extended infra soft compactness (resp., extended infra soft Lindelöfness) of $(\mathscr{A}, \Omega, \mathscr{B})$ implies that $\mathscr{B}$ is finite (resp., countable).

In the next result, we determine under which conditions the converse of Theorem 45 hold.
Theorem 47. Let $(\mathscr{A}, \Omega, \mathscr{B})$ be extended such that $\mathscr{B}$ is finite (resp., countable). Then, $(\mathscr{A}, \Omega, \mathscr{B})$ is infra soft compact (resp., extended infra soft Lindelöf) space iff $\left(\mathscr{A}, \Omega_{\beta}\right)$ is infra compact (resp., infra Lindelöf) for each $\beta \in \mathscr{B}$.

Proof. The necessary part was proved in Theorem 45. To prove the sufficient part, consider $\Theta=\left\{\left(\delta_{k}, \mathscr{B}\right): k \in K\right\}$ as an infra soft open cover of $(\mathscr{A}, \Omega, \mathscr{B})$. Then, $\mathscr{A}=\bigcup_{k \in K} \delta_{k}(\beta)$ for each $\beta \in \mathscr{B}$. By hypothesis of infra compactness (resp., resp., infra Lindelöfness), we obtain $\mathscr{A}=\bigcup_{k \in I} \delta_{k}(\beta)$ for each $\beta \in \mathscr{B}$, where $I$ is finite (resp., countable). Now, we take from $\Theta$ all infra soft open sets which (at least) one of their $\beta$-components belong to $\left\{\delta_{k}(\beta): k \in I\right\}$. Hence, we obtain the desired result.

Proposition 48. Let $(\mathscr{A}, \Omega, \mathscr{B})$ be stable. Then, $(\mathscr{A}, \Omega, \mathscr{B})$ is infra soft compact (resp., infra soft Lindelöf) space iff $\left(\mathscr{A}, \Omega_{\beta}\right)$ is infra compact (resp., infra Lindelöf).

Proof. Straightforward.

Theorem 49. $(\xi, \mathscr{B})$ is infra soft compact (resp., infra soft Lindelöf) subset of $(\mathscr{A}, \Omega, \mathscr{B})$ iff a soft subspace $((\xi, \mathscr{B})$, $\left.\Omega_{(\xi, \mathscr{B})}, \mathscr{B}\right)$ is infra soft compact (resp., infra soft Lindelöf).

Proof. Necessity: consider $\left\{\left(\delta_{k}, \mathscr{B}\right): k \in K\right\}$ as an infra soft open cover of $\left((\xi, \mathscr{B}), \Omega_{(\xi, \mathscr{B})}, \mathscr{B}\right)$. Then, for each $\left(\delta_{k}, \mathscr{B}\right) \epsilon$ $\Omega_{(\xi, \mathscr{B})}$, there exists $\left(\pi_{k}, \mathscr{B}\right) \in \Omega$ such that $\left(\delta_{k}, \mathscr{B}\right)=\left(\pi_{k}, \mathscr{B}\right)$ $\bigcap^{\sim}(\xi, \mathscr{B})$. Now, $\left\{\left(\pi_{k}, \mathscr{B}\right): k \in K\right\}$ is an infra soft open cover of $(\xi, \mathscr{B})$. By hypothesis, $(\xi, \mathscr{B}) \subseteq \sim \bigcup^{\sim n}{ }_{k=1}^{n}\left(\pi_{k}, \mathscr{B}\right)$. Therefore, $(\xi, \mathscr{B}) \subseteq \sim \bigcup^{\sim n}{ }_{k=1}^{n}\left[\left(\pi_{k}, \mathscr{B}\right) \bigcap^{\sim}(\xi, \mathscr{B})\right]=\bigcup_{k=1}^{\sim n}\left(\delta_{k}, \mathscr{B}\right) . \quad$ Thus, $\left((\xi, \mathscr{B}), \Omega_{(\xi, \mathscr{B})}, \mathscr{B}\right)$ is infra soft compact.

Sufficiency: consider $\left\{\left(\delta_{k}, \mathscr{B}\right) \in \Omega: k \in K\right\}$ as an infra soft open cover of $(\xi, \mathscr{B})$. Then, $\left\{\left(\delta_{k}, \mathscr{B}\right) \bigcap^{\sim}(\xi, \mathscr{B}): k \in K\right\}$ is an infra soft open cover of $\left((\xi, \mathscr{B}), \Omega_{(\xi, \mathscr{B})}, \mathscr{B}\right)$. By hypothesis, $(\xi, \mathscr{B}) \subseteq \sim \bigcup^{\sim}{ }_{k=1}^{n}\left[\left(\delta_{k}, \mathscr{B}\right) \bigcap^{\sim}(\xi, \mathscr{B})\right]$. Therefore, $(\xi, \mathscr{B})$ $\subseteq \sim \bigcup_{k=1}^{\sim n}\left(\delta_{k}, \mathscr{B}\right)$. Thus, $(\xi, \mathscr{B})$ is infra soft compact.

Following similar arguments, one can prove the case between parentheses.

Theorem 50. The finite product of infra soft compact (resp., infra soft Lindelöf) spaces is infra soft compact (resp., infra soft Lindelöf).

Proof. Let $\left(\mathscr{A}_{1}, \Omega_{1}, \mathscr{B}_{1}\right)$ and $\left(\mathscr{A}_{2}, \Omega_{2}, \mathscr{B}_{2}\right)$ be two infra soft Lindelöf spaces. Consider $\left\{\left(\xi_{k}, \mathscr{B}_{1} \times \mathscr{B}_{2}\right): k \in K\right\}$ as an infra soft open cover of $\widetilde{\mathscr{A}}_{1} \times \widetilde{\mathscr{A}}_{2}$. Now, for each $k \in K$ $\left(\xi_{k}, \mathscr{B}_{1} \times \mathscr{B}_{2}\right)=\left(\delta_{k}, \mathscr{B}_{1}\right) \times\left(\lambda_{k}, \mathscr{B}_{2}\right)$, where $\left(\delta_{k}, \mathscr{B}_{1}\right) \in \Omega_{1}$ and $\left(\lambda_{k}, \mathscr{B}_{2}\right) \in \Omega_{2}$. Then $\widetilde{\mathscr{A}}_{1} \times \widetilde{\mathscr{A}}_{2}=\bigcup^{\sim}{ }_{k \in K}\left(\xi_{k}, \mathscr{B}_{1} \times \mathscr{B}_{2}\right)$ $\subseteq \sim\left[\cup^{\sim}{ }_{k \in K}\left(\delta_{k}, \mathscr{B}_{1}\right)\right] \times\left[\bigcup_{k \in K}^{\sim}\left(\lambda_{k}, \mathscr{B}_{2}\right)\right]$. That is, $\widetilde{\mathscr{A}}_{1}=$ $\bigcup_{k \in K}^{\sim}\left(\delta_{k}, \mathscr{B}_{1}\right)$ and $\widetilde{\mathscr{A}}_{2}=\bigcup^{\sim}{ }_{k \in K}\left(\lambda_{k}, \mathscr{B}_{2}\right)$. By hypothesis, there are two countable sets $I$ and $J$ such that $\widetilde{\mathscr{A}}_{1}=$ $\bigcup_{i \in I}^{\sim}\left(\delta_{i}, \mathscr{B}_{1}\right)$ and $\widetilde{\mathscr{A}}_{2}=\bigcup^{\sim} \in J\left(\lambda_{j}, \mathscr{B}_{2}\right)$. Thus, $\widetilde{\mathscr{A}}_{1} \times \widetilde{\mathscr{A}}_{2}=$ $\left[\cup_{i \in I}^{\sim}\left(\delta_{i}, \mathscr{B}_{1}\right)\right] \times\left[\bigcup_{j \in J}^{\sim}\left(\lambda_{j}, \mathscr{B}_{2}\right)\right]=\bigcup_{k \in I}^{\sim}\left(\xi_{k}, \mathscr{B}_{1} \times \mathscr{B}_{2}\right)$. Since $I \cup J$ is a countable set, we obtain the desired result. 
Following similar arguments, one can prove the theorem in the case of infra soft compact.

Theorem 51. Every uncountable (resp., infinite) subset of an infra soft Lindelöf (resp., infra soft compact) space has an infra soft limit point.

Proof. Let $(\delta, \mathscr{B})$ be an uncountable subset of $(\mathscr{A}, \Omega, \mathscr{B})$ which is an infra soft Lindelöf space. Suppose that no soft point of $\tilde{\mathscr{A}}$ is an infra soft limit point of $(\delta, \mathscr{B})$. Then for each $P_{\beta}^{\alpha} \in \tilde{\mathscr{A}}$, there is an infra soft open set $\left(\lambda_{\alpha_{k}}, \mathscr{B}\right)$ containing $P_{\beta}^{\alpha}$ such that $\left(\lambda_{\alpha_{k}}, \mathscr{B}\right) \bigcap^{\sim}(\delta, \mathscr{B}) \backslash P_{\beta}^{\alpha}=\Phi$. Now, a family $\Lambda=\left\{\left(\lambda_{\alpha_{k}}, \mathscr{B}\right): k \in K\right\}$ forms an infra soft open cover of $\tilde{\mathscr{A}}$. Since $\mathscr{A}$ is infra soft Lindelöf, there is a countable set $I$ such that $\tilde{\mathscr{A}}=\bigcup_{k \in I}^{\sim}\left(\lambda_{\alpha_{k}}, \mathscr{B}\right)$. Therefore, $\mathscr{A}$ has at most countable soft points of $(\delta, \mathscr{B})$. This means that $(\delta, \mathscr{B})$ is countable which contradicts the uncountability of $(\delta, \mathscr{B})$. Hence, $(\delta$, $\mathscr{B})$ has an infra soft limit point.

Following similar arguments, one can prove the case between parentheses.

\section{Fixed Point Theorem in Infra Soft Topological Spaces}

Through this portion, we aim to introduce the concept of fixed soft points in the frame of ISTSs and establish its master properties. We present interesting findings that associated fixed soft points with infra soft compact spaces. Finally, the transmission of fixed soft points from infra soft topology to classical infra topology and vice versa is investigated.

Theorem 52. Let $\left\{\mathscr{C}_{n}: n \in \mathbb{N}\right\}$ be a family of soft sets in an infra soft compact space $(\mathscr{A}, \Omega, \mathscr{B})$. Then $\bigcap_{n \in \mathbb{N}}^{\sim} \mathscr{C}_{n} \neq \tilde{\Phi}$ provided that the following three conditions are satisfied:

(i) $\mathscr{C}_{n} \neq \tilde{\Phi}$ for each $n \in \mathbb{N}$

(ii) $\mathscr{C}_{n}$ is an infra soft closed set for each $n \in \mathbb{N}$

(iii) $\mathscr{C}_{n+1}$ is a subset of $\mathscr{C}_{n}$ for each $n \in \mathbb{N}$

Proof. Suppose that $\bigcap_{n \in \mathbb{N}}^{\sim} \mathscr{C}_{n}=\tilde{\Phi}$. Then, $\bigcup_{n \in \mathbb{N}}^{\sim} \mathscr{C}_{n}^{c}=\tilde{\mathscr{A}}$. According to (ii), we obtain $\left\{\mathscr{C}_{n}^{c}: n \in \mathbb{N}\right\}$ forms an infra soft open cover of $\tilde{\mathcal{A}}$. By the hypothesis of infra soft compactness, there exist $k_{1}, k_{2}, \cdots, k_{j} \in \mathbb{N}, \quad k_{1}<k_{2}<\cdots<k_{j}$ such that $\tilde{\mathscr{A}}=\mathscr{C}_{k_{1}}^{c} \bigcup^{\sim} \mathscr{C}_{k_{2}}^{c} \bigcup^{\sim} \cdots \cup^{\sim} \mathscr{C}_{k_{j}}^{c}$. It comes from (iii) that $\mathscr{C}_{k_{j}}$ $\subseteq^{\sim} \tilde{A}=\mathscr{C}_{k_{1}}^{c} \bigcup^{\sim} \mathscr{C}_{k_{2}}^{c} \bigcup^{\sim} \cdots \cup^{\sim} \mathscr{C}_{k_{j}}^{c}=\left[\mathscr{C}_{k_{1}} \bigcap^{\sim} \mathscr{C}_{k_{2}} \cap^{\sim} \cdots \bigcap^{\sim} \mathscr{C}_{k_{j}}\right]^{c}$ $=\mathscr{C}_{k_{j}}^{c}$. This is a contradiction. Hence, $\bigcap_{n \in \mathbb{N}}^{\sim} \mathscr{C}_{n} \neq \tilde{\Phi}$, as required.

Proposition 53. Let $(\mathscr{A}, \Omega, \mathscr{B})$ be an infra soft compact and infra soft $T_{2}$-space such that $\Omega$ is closed under finite union. If $f_{\varphi}:(\mathscr{A}, \Omega, \mathscr{B}) \longrightarrow(\mathscr{A}, \Omega, \mathscr{B})$ is infra soft continuous, then $f_{\varphi}$ has a unique soft point $P_{\beta}^{\alpha} \in \tilde{\mathscr{A}}$.
Proof. Let $\left\{\mathscr{C}_{n}=f_{\varphi}\left(\mathscr{C}_{n-1}\right)=f_{\varphi}^{n}(\tilde{\mathscr{A}})\right.$ for each $n \in \mathbb{N}$, where $\left.\mathscr{C}_{1}=f_{\varphi}(\tilde{\mathscr{A}})\right\}$ be a family of soft sets in $(\mathscr{A}, \Omega, \mathscr{B})$. Obviously, $\mathscr{C}_{n+1} \subseteq \sim \mathscr{C}_{n}$ for each $n \in \mathbb{N}$. Since $f_{\varphi}$ is an infra soft continuous map, $\mathscr{C}_{n}$ is an infra soft compact set for each $n \in \mathbb{N}$, and since $(\mathscr{A}, \Omega, \mathscr{B})$ is an infra soft $T_{2}$-space such that $\Omega$ is closed under finite union, $\mathscr{C}_{n}$ is an infra soft closed set for each $n$ $\in \mathbb{N}$. Putting $(\delta, \mathscr{B})=\bigcap^{\sim}{ }_{n \in \mathbb{N}} \mathscr{C}_{n}$, according to Theorem 52, we find $(\delta, \mathscr{B})$ is a nonnull soft set. Note that $f_{\varphi}(\delta, \mathscr{B})=f_{\varphi}$ $\left(\bigcap_{n \in \mathbb{N}}^{\sim} f_{\varphi}^{n}\left(\mathscr{A}^{\sim}\right)\right) \simeq^{\sim} \bigcap_{n \in \mathbb{N}}^{\sim} f_{\varphi}^{n+1}\left(\mathscr{A}^{\sim}\right) \subseteq \sim \bigcap^{\sim}{ }_{n \in \mathbb{N}} f_{\varphi}^{n}(\tilde{\mathscr{A}})=(\delta$, $\mathscr{B})$. To prove that $(\delta, \mathscr{B}) \subseteq \sim f_{\varphi}(\delta, \mathscr{B})$, suppose that there exists a $P_{\beta}^{\alpha} \in(\delta, \mathscr{B})$ such that $P_{\beta}^{\alpha} \in f_{\varphi}(\delta, \mathscr{B})$. Let $\mathscr{D}_{n}=f_{\varphi}^{-1}\left(P_{\beta}^{\alpha}\right)$ $\cap^{\sim} \mathscr{C}_{n}$. It is clear that $\mathscr{D}_{n} \neq \tilde{\Phi}$ and $\mathscr{D}_{n} \subseteq \sim \mathscr{D}_{n-1}$ for each $n$ $\in \mathbb{N}$. Now, $\mathscr{D}_{n}$ is an infra soft closed set for each $n \in \mathbb{N}$, and by Theorem 52, there is a soft point $P_{\rho}^{\gamma}$ such that $P_{\rho}^{\gamma} \in f_{\varphi}^{-1}\left(P_{\beta}^{\alpha}\right) \bigcap^{\sim} \mathscr{C}_{n}$. Therefore, $P_{\beta}^{\alpha}=f_{\varphi}\left(P_{\rho}^{\gamma}\right) \in f_{\varphi}(\delta, \mathscr{B})$; this is a contradiction. Hence, $f_{\varphi}(\delta, \mathscr{B})=(\delta, \mathscr{B})$ which means we obtain the desired result.

Definition 54 . An ISTS $(\mathscr{A}, \Omega, \mathscr{B})$ is said to have a fixed soft point property if every infra soft continuous map $f_{\varphi}:(\mathscr{A}$, $\Omega, \mathscr{B}) \longrightarrow(\mathscr{A}, \Omega, \mathscr{B})$ has a fixed soft point, i.e., there exists $P_{\beta}^{\alpha} \in \mathscr{A}$ such that $f_{\varphi}\left(P_{\beta}^{\alpha}\right)=P_{\beta}^{\alpha}$.

Proposition 55. The property of being a fixed soft point is an infra soft topological property.

Proof. Let $\left(\mathscr{A}_{1}, \Omega_{1}, \mathscr{B}_{1}\right)$ and $\left(\mathscr{A}_{2}, \Omega_{2}, \mathscr{B}_{2}\right)$ be an infra soft homeomorphism. Then there is a bijective soft map $f_{\varphi}:\left(\mathscr{A}_{1}\right.$, $\left.\Omega_{1}, \mathscr{B}_{1}\right) \longrightarrow\left(\mathscr{A}_{2}, \Omega_{2}, \mathscr{B}_{2}\right)$ such that $f_{\varphi}$ and $f_{\varphi}^{-1}$ are infra soft continuous. Consider that $\left(\mathscr{A}_{1}, \Omega_{1}, \mathscr{B}_{1}\right)$ has a fixed soft point property, i.e., every infra soft continuous map $f_{\varphi}:\left(\mathscr{A}_{1}, \Omega_{1}\right.$, $\left.\mathscr{B}_{1}\right) \longrightarrow\left(\mathscr{A}_{1}, \Omega_{1}, \mathscr{B}_{1}\right)$ has a fixed soft point. Now, let $h_{\varphi}$ : $\left(\mathscr{A}_{2}, \Omega_{2}, \mathscr{B}_{2}\right) \longrightarrow\left(\mathscr{A}_{2}, \Omega_{2}, \mathscr{B}_{2}\right)$ be an infra soft continuous map. Obviously, $h_{\varphi} \circ f_{\varphi}:\left(\mathscr{A}_{1}, \Omega_{1}, \mathscr{B}_{1}\right) \longrightarrow\left(\mathscr{A}_{2}, \Omega_{2}, \mathscr{B}_{2}\right)$ is an infra soft continuous map. Also, $f_{\varphi}^{-1} \circ h_{\varphi} \circ f_{\varphi}:\left(\mathscr{A}_{1}, \Omega_{1}\right.$, $\left.\mathscr{B}_{1}\right) \longrightarrow\left(\mathscr{A}_{1}, \Omega_{1}, \mathscr{B}_{1}\right)$ is an infra soft continuous. Since $\left(\mathscr{A}_{1}\right.$ , $\left.\Omega_{1}, \mathscr{B}_{1}\right)$ has a fixed soft point property, $f_{\varphi}^{-1}\left(h_{\varphi}\left(f_{\varphi}\left(P_{\beta}^{\alpha}\right)\right)\right)=$ $P_{\beta}^{\alpha}$ for some $P_{\beta}^{\alpha} \in \tilde{A}$, so $f_{\varphi}\left(f_{\varphi}^{-1}\left(h_{\varphi}\left(f_{\varphi}\left(P_{\beta}^{\alpha}\right)\right)\right)\right)=f_{\varphi}\left(P_{\beta}^{\alpha}\right)$. This leads to $h_{\varphi}\left(f_{\varphi}\left(P_{\beta}^{\alpha}\right)\right)=f_{\varphi}\left(P_{\beta}^{\alpha}\right)$. Thus, $f_{\varphi}\left(P_{\beta}^{\alpha}\right)$ is a fixed soft point of $h_{\varphi}$. This ends the proof that $\left(\mathscr{A}_{2}, \Omega_{2}, \mathscr{B}_{2}\right)$ has a fixed soft point property.

Theorem 56. Consider $\Omega$ as an extended infra soft topology over $\mathscr{A}_{1}$. A soft map $f_{\varphi}:\left(\mathscr{A}_{1}, \Omega, \mathscr{B}_{1}\right) \longrightarrow\left(\mathscr{A}_{2}, \mho, \mathscr{B}_{2}\right)$ is infra soft continuous iff a crisp map $f:\left(\mathscr{A}_{1}, \Omega_{\beta}\right) \longrightarrow\left(\mathscr{A}_{2}\right.$, $\left.\mho_{\varphi(\beta)}\right)$ is infra continuous.

Proof. [ $\Rightarrow]$ : let $H$ be an infra open set in $\left(\mathscr{A}_{2}, \mho_{\varphi(\beta)}\right)$. Then there is an infra soft open set $\left(\delta, \mathscr{B}_{2}\right)$ in $\left(\mathscr{A}_{2}, \mho, \mathscr{B}_{2}\right)$ such that $\delta(\varphi(\beta))=H$. Since $f_{\varphi}$ is an infra soft continuous map, $f_{\varphi}^{-1}\left(\delta, \mathscr{B}_{2}\right)$ is an infra soft open set. Therefore, a soft set $f_{\varphi}^{-1}$ $\left(\delta, \mathscr{B}_{2}\right)=\left(f^{-1}(\delta), \mathscr{B}_{1}\right)$ in $\left(\mathscr{A}_{1}, \Omega, \mathscr{B}_{1}\right)$ is given by $f_{\varphi}^{-1}(\delta)(\beta)$ 
$=f^{-1}(\delta(\varphi(\beta)))$ for each $\beta \in \mathscr{B}_{1}$. By hypothesis, $\Omega$ is extended, a set $f^{-1}(\delta(\varphi(\beta)))=f^{-1}(H)$ in $\left(\mathscr{A}_{1}, \Omega_{\beta}\right)$ is infra open. Hence, $f$ is an infra continuous map.

$[\Leftarrow]$ : let $\left(\xi, \mathscr{B}_{2}\right)$ be an infra soft open set in $\left(\mathscr{A}_{2}, \mho, \mathscr{B}_{2}\right)$. Then a soft set $f_{\phi}^{-1}\left(\xi, \mathscr{B}_{2}\right)=\left(f_{\varphi}^{-1}(\xi), \mathscr{B}_{1}\right)$ in $\left(\mathscr{A}_{1}, \Omega, \mathscr{B}_{1}\right)$ is given by $f_{\varphi}^{-1}(\xi)(\beta)=f^{-1}(\xi(\varphi(\beta)))$ for each $\beta \in \mathscr{B}_{1}$. Since $f$ is an infra continuous map, a subset $f^{-1}(\xi(\varphi(\beta)))$ in $\left(\mathscr{A}_{1}\right.$, $\left.\Omega_{\beta}\right)$ is infra open. By hypothesis, $\Omega$ is extended, we obtain $f_{\varphi}^{-1}\left(\xi, \mathscr{B}_{2}\right)$ is an infra soft open set in $\left(\mathscr{A}_{1}, \Omega, \mathscr{B}_{1}\right)$. Hence, $f_{\varphi}$ is an infra soft continuous map.

Proposition 57. If an ISTS $(\mathscr{A}, \Omega, \mathscr{B})$ has a fixed soft point, then $\left(\mathscr{A}, \Omega_{\beta}\right)$ has a fixed point property for all $\beta \in \mathscr{B}$.

Proof. Consider an ISTS $(\mathscr{A}, \Omega, \mathscr{B})$ as a fixed soft point property. This means that any infra soft continuous map $f_{\varphi}:(\mathscr{A}$, $\Omega, \mathscr{B}) \longrightarrow(\mathscr{A}, \Omega, \mathscr{B})$ has a fixed soft point. Say, $P_{\beta}^{\alpha}$. It comes from Theorem 56 that $f:\left(\mathscr{A}, \Omega_{\beta}\right) \longrightarrow\left(\mathscr{A}, \Omega_{\varphi(\beta)}\right)$ is infra continuous. Since $P_{\beta}^{\alpha}$ is a fixed soft point of $f_{\varphi}$, we obtain $f(\alpha)=\alpha$. Thus, $f$ has a fixed point.

\section{Conclusion and Future Work}

This article is aimed at completely presenting and scrutinizing the concepts and notions in the frame of ISTSs. So, we have initiated two sorts of covering properties in the frame of ISTSs which consider two classifications of soft spaces. Also, we have established the concept of fixed soft points and elucidated essential properties, in particular, those induced from infra soft compact spaces.

We sum our accomplishments through this work in the following:

(1) Introduce the concepts of infra soft compact and infra soft Lindelöf spaces

(2) Offer some illustrative examples to point out the relationships between these two spaces and validate the obtained findings and notes

(3) Explain the interchangeable property of these concepts from infra soft topology to classical infra topology and vice versa

(4) Investigate these concepts under finite product of soft spaces and infra soft homeomorphism

(5) Display the concept of fixed soft points and reveal its basic features

Our blueprint in the forthcoming papers is as follows.

(1) Establish new sorts of covering properties like almost infra soft compact (Lindelöf) and nearly infra soft compact (Lindelöf) spaces

(2) Investigate metric spaces in the content of ISTSs which will open a door for more studies on fixed soft points
(3) Carry out further investigations in the areas of rough set theory and infra topologies

(4) Generalize ISTSs to infra soft bitopological and infra soft ordered topological spaces

\section{Data Availability}

No data were used to support this study.

\section{Conflicts of Interest}

The author declares no conflicts of interest.

\section{References}

[1] D. Molodtsov, "Soft set theory-first results," Computers \& Mathematics with Applications, vol. 37, no. 4-5, pp. 19-31, 1999.

[2] S. Yuksel, T. Dizman, G. Yildizdan, and U. Sert, "Application of soft sets to diagnose the prostate cancer risk," Journal of Inequalities and Applications, vol. 2013, no. 1, 2013.

[3] N. Çağman and S. Enginoğlu, "Soft matrix theory and its decision making," Computers and Mathematics with Applications, vol. 59, no. 10, pp. 3308-3314, 2010.

[4] T. M. Al-shami and M. E. El-Shafei, "Partial belong relation on soft separation axioms and decision-making problem, two birds with one stone," Soft Computing, vol. 24, no. 7, pp. 5377-5387, 2020.

[5] P. K. Maji, R. Biswas, and R. Roy, "Soft set theory," Computers \& Mathematics with Applications, vol. 45, no. 4-5, pp. 555562, 2003.

[6] M. I. Ali, F. Feng, X. Liu, W. K. Min, and M. Shabir, "On some new operations in soft set theory," Computers and Mathematics with Applications, vol. 57, no. 9, pp. 1547-1553, 2009.

[7] K. Qin and Z. Hong, "On soft equality," Journal of Computational and Applied Mathematics, vol. 234, no. 5, pp. 13471355, 2010.

[8] M. Abbas, M. I. Ali, and S. Romaguera, "Generalized operations in soft set theory via relaxed conditions on parameters," Univerzitet $u$ Nišu, vol. 31, no. 19, pp. 5955-5964, 2017.

[9] T. M. Al-shami and M. E. El-Shafei, "T-soft equality relationTsoft equality relation," Turkish Journal of Mathematics, vol. 44, no. 4, pp. 1427-1441, 2020.

[10] M. Shabir and M. Naz, "On soft topological spaces," Computers and Mathematics with Applications, vol. 61, no. 7, pp. 1786-1799, 2011.

[11] N. Çağman, S. Karataş, and S. Enginoglu, "Soft topology," Computers and Mathematics with Applications, vol. 62, no. 1, pp. 351-358, 2011.

[12] S. Nazmul and S. K. Samanta, "Neighbourhood properties of soft topological spaces," Annals of Fuzzy Mathematics and Informatics, vol. 6, no. 1, pp. 1-15, 2013.

[13] T. M. Al-shami and L. D. R. Kocinac, "The equivalence between the enriched and extended soft topologies," Applied and Computational Mathematics, vol. 18, no. 2, pp. 149-162, 2019.

[14] S. A. El-Sheikh and A. M. Abd El-Latif, "Decompositions of some types of supra soft sets and soft continuity," International Journal of Mathematics Trends and Technology, vol. 9, no. 1, pp. 37-56, 2014. 
[15] B. M. Ittanagi, "Soft bitopological spaces," International Journal of Computer Applications, vol. 107, no. 7, pp. 1-4, 2014.

[16] T. M. Al-shami, "New soft structure: infra soft topological spaces," Mathematical Problems in Engineering, vol. 2021, Article ID 3361604, p. 12, 2021.

[17] A. Azzam, A. M. Khalil, and S.-G. Li, "Medical applications via minimal topological structure," Journal of Intelligent and Fuzzy Systems, vol. 39, no. 3, pp. 4723-4730, 2020.

[18] M. M. El-Sharkasy, "Minimal structure approximation space and some of its application," Journal of Intelligent and Fuzzy Systems, vol. 40, no. 1, pp. 973-982, 2021.

[19] T. M. Al-shami, M. E. El-Shafei, and M. Abo-Elhamayel, "Almost soft compact and approximately soft Lindelöf spaces," Journal of Taibah University for Science, vol. 12, no. 5, pp. 620-630, 2018.

[20] A. Aygünoğlu and H. Aygün, "Some notes on soft topological spaces," Neural Computing and Applications, vol. 21, pp. 113119, 2012.

[21] T. Hida, "A comprasion of two formulations of soft compactness," Annals of Fuzzy Mathematics and Informatics, vol. 8, no. 4, pp. 511-524, 2014.

[22] N. Alamgir, Q. Kiran, H. Isk, and H. Aydi, "Fixed point results via a Hausdorff controlled type metric," Advances in Difference Equations, vol. 2020, no. 1, 2020.

[23] T. M. Al-shami and E. A. Abo-Tabl, "Soft $\alpha$-separation axioms and $\alpha$-fixed soft points," AIMS Mathematics, vol. 6, no. 6, pp. 5675-5694, 2021.

[24] A. Asif, N. Hussain, H. Al-Sulami, and M. Arshad, "Some fixed point results in function weighted metric spaces," Journal of Mathematics, vol. 2021, Article ID 6636504, 9 pages, 2021.

[25] H. Işık, V. Parvaneh, and M. R. Haddadi, "Strong and pure fixed point properties of mappings on normed spaces," Mathematical Sciences, 2021.

[26] S. Das and S. K. Samanta, "Soft metric," Annals of Fuzzy Mathematics and Informatics, vol. 6, no. 1, pp. 77-94, 2013.

[27] M. E. El-Shafei, M. Abo-Elhamayel, and T. M. Al-shami, "Partial soft separation axioms and soft compact spaces," Univerzitet $u$ Nišu, vol. 32, no. 13, pp. 4755-4771, 2018.

[28] F. Feng, Y. M. Li, B. Davvaz, and M. I. Ali, "Soft sets combined with fuzzy sets and rough sets: a tentative approach," Soft Computing, vol. 14, no. 9, pp. 899-911, 2010.

[29] A. Kharal and B. Ahmed, "Mappings on soft classes," New Mathematic Natural Computing, vol. 7, no. 3, pp. 471-481, 2011.

[30] I. Zorlutuna, M. Akdag, W. K. Min, and S. Atmaca, "Remarks on soft topological spaces," Annals of Fuzzy Mathematics and Informatics, vol. 2, pp. 171-185, 2012.

[31] T. M. Al-shami and I. Alshammari, Infra Soft Quotient Mappings, 2021. 